\title{
Género y crianza en Lima: los niños y las niñas del distrito de San Juan de Lurigancho, Lima
}

\author{
José Antonio Panduro \\ Universidad Nacional Federico Villarreal \\ Lima, Perú
}

Recibido: 3 de mayo del 2016 / Aprobado: 19 de julio del 2016

Las desigualdades de género son un aspecto importante por resolver si se pretende una mejoría en los indicadores del desarrollo humano de la sociedad. Intentando conocer si estas diferencias se originan en la crianza desde las acciones concretas en el vínculo con los niños, fueron evaluadas 241 diadas madre-niño de un centro educativo inicial del distrito de San Juan de Lurigancho (Lima), en las cuales los hijos fueron 119 niños y 122 niñas cuyas edades fluctuaron entre los 4 y 7 años. Las mediciones de autorreporte se obtuvieron de la Escala de Evaluación de la Estimulación Infantil en las Relaciones Madre-Niño: Escala EEI (Panduro, 1998a), la cual considera la promoción y el control conductual en el vínculo como las dimensiones fundamentales de la crianza. Para efectos del estudio, se realizaron análisis factoriales para identificar los componentes al interior de cada dimensión de la crianza. Los resultados señalan, en general, condiciones similares experimentadas tanto por niños como por niñas; sin embargo, en el componente Mediación de la Socialización, ellos obtuvieron niveles significativamente mayores que ellas $(p<.05)$.

Desigualdades de género / análisis factorial / orientación al logro

\section{Gender and Child Rearing in Lima: the Boys and Girls from the District of San Juan de Lurigancho, Lima}

Gender inequality constitutes a relevant problem for the improvement of the indicators of human development in society. To this end, we examined 241 mother-child dyads in an elementary school in the district of San Juan de Lurigancho, in the city of Lima. The sample was comprised of 119 boys and 122 girls, aged 7 to 9 years. Measurements were obtained through the Mother-Child Relationship Stimulation Scale (Panduro, 1998a) in a self-report. To identify the different components of child rearing, we applied factorial analyses. Results show that boys and girls have similar rearing conditions; however, in Socialization Mediation, boys obtain a higher value $(p<.05)$.

gender inequalities / factor analysis / results oriented attitude

Correo electrónico: joseantoniopanduro@outlook.com.pe 


\section{INTRODUCCIÓN}

Según el INEI (2014), en el Perú, el 76 \% de las personas analfabetas es mujer, sus ingresos son $30 \%$ inferiores a los de los hombres, y son el $87 \%$ de las víctimas de violencia familiar y sexual. En el año 2013, del total de víctimas de abuso sexual, el $93 \%$ fue una mujer menor de 18 años.

Además de estas críticas estadísticas, la desigualdad entre mujeres y varones en el Perú, con desventaja para las niñas, también se expresa en aspectos como una mayor tasa de deserción escolar y menores logros en el aprendizaje, así como en una sobrecarga de responsabilidades en el hogar, que incluyen muchas veces el cuidado de otros menores como ellas, entre otras oportunidades más restringidas de desarrollo por ser niñas.

La escuela suele reforzar estereotipos de exclusión al establecer desproporciones en las responsabilidades y la participación en ambientes como el aula y el patio de recreo, que reiteran la invisibilidad y el menor protagonismo, y promueven mensajes que asocian la limpieza y cocina con las mujeres mientras las actividades intelectuales, o que implican despliegue de fortaleza y liderazgo, son vinculadas con los varones. Así se estimula la timidez y contención en ellas, y la expresividad y el activismo en ellos (Unicef, 2014).

Una de las formas de medir estas brechas es el Índice de Desigualdad de Género (Gender Inequality Index o GII), índice que compila logros en salud reproductiva (tasa de mortalidad materna y tasa de fecundidad adolescente), empoderamiento (número de mujeres con escaños en el Congreso y población con, al menos, educación secundaria completa) y presencia laboral (tasa de participación en la fuerza de trabajo). Los valores más altos en el GII indican más desigualdades de género $\mathrm{y}$, por lo tanto, una mayor pérdida para el desarrollo humano.

El Índice de Desigualdad de Género en el Perú es 0.387 y la salud reproductiva es el factor que más contribuye con él, ya que en nuestro país la mortalidad materna es elevada, 67 muertes maternas por cada 100000 nacidos vivos (PNUD, 2014).

Desde una visión global, el GII del Perú lo ubica en el puesto 77 de 149 países en esta medición. Los dos países con GII más bajos son Eslovenia (0.021) y Suiza (0.030); los dos más altos, Yemén (0.733) y Chad (0.707).

Estas diferencias se van construyendo desde lo cotidiano en el hogar a través de las actitudes y acciones de los padres y familiares: la madre es quien tiene una influencia más significativa. Esta diferenciación se expresa a través de expectativas pobres o de fracaso hacia las niñas, manifestadas en la distribución desproporcional de las responsabilidades del hogar; en mensajes que sostienen que ellas deben depender de un hombre para lograr su potencial, estar seguras o ser felices; que su rol debe circunscribirse a cuidar y servir a los varones del hogar. En esta visión, los niños y varones adultos, más bien, deben ser considerados merecedores de mejores oportunidades de entretenimiento, educación y mayores 
libertades en comparación con las mujeres; su rol en los vínculos que establezcan debe orientarse a producir y demostrar fortaleza. Una de las más complejas desigualdades que experimentan las niñas es la educación en el silencio, es decir, que las niñas y las mujeres deban tener limitado su derecho a expresar insatisfacciones, quejarse, protestar o enfurecerse, pues en ese rumbo pueden perder su feminidad o constituirse en amenaza para los varones.

Como se observa, estas diferencias entre niños y niñas comienzan desde muy temprano; muchas de ellas se amplifican progresivamente en el tiempo y se constituyen en una pauta natural en la adultez.

Cuando los niños son pequeños, el ambiente en el que se desenvuelven se halla estructurado por su grupo familiar. Dentro de él, la figura que evidentemente adopta mayor relevancia, al menos en nuestra cultura, es la de sus madres, las cuales constituyen para ellos los primeros y principales focos de estimulación durante los primeros años de sus desarrollos.

La familia tiene una significativa influencia en el desarrollo del niño en un sentido amplio; por ejemplo, sobre el temperamento y ajuste socioemocional en la infancia (Ato, Galián, y Huéscar, 2007) o sobre el desarrollo de competencias sociales (Berk, 2004), entre otros.

Henao, Ramírez y Ramírez (2007), por su parte, plantean la importancia de la familia en la socialización y desarrollo durante la infancia; precisan que la combinación de costumbres y hábitos de crianza de los padres, la sensibilidad hacia las necesidades de su hijo, la aceptación de su individualidad, así como el afecto expresado y los mecanismos de control desplegados, resultan fundamentales para regular su comportamiento.

No ha sido sino hasta hace unas cinco décadas que se han iniciado estudios sistemáticos en torno a la importancia que tiene la relación madre-niño sobre la conducta y el desarrollo de este. Una perspectiva para dar cuenta de ello es aquella que estudia la influencia de las óptimas interacciones madre-niño sobre múltiples aspectos positivos en el desarrollo del niño; por ejemplo, sobre el desarrollo de habilidades de comunicación (Flanagan, Coppa, Riggs, y Alario, 1994), competencias lingüísticas y sociales (Musselman y Churchill, 1991), habilidades socioemocionales (Denham, 1993) o cognitivas (Esser, Dinter, Jorg, Rose, Villalba, Laucht, y Schmidt, 1993). Asimismo, se ha identificado que la calidez en la relación madre-niño es un factor que modera la relación entre autoestima y las habilidades intrínsecas a la teoría de la mente (Cahill, Deater-Deckard, Pike, y Hughes, 2007), y que el vínculo que establece el niño con su madre tiene impacto sobre el desarrollo de la conciencia, entendida esta como la emoción, conducta y cognición moral, elementos que permiten la adquisición de los valores familiares y culturales (Kochanska, Forman, Aksan y Dunbar, 2005).

Por otro lado, una línea de investigación que resulta ser muy prolija es aquella que estudia cómo los trastornos psicológicos y emocionales, así como determinadas actitudes en la madre, alteran el desarrollo o limitan el aprendizaje de múltiples habilidades en los niños. 
En general, el desarrollo de un niño depende mucho del número y de la calidad de oportunidades que encuentre disponibles en su interacción con las personas, en especial los padres, quienes se constituyen en el principal objeto de estimulación social durante su infancia (Bijou y Baer, 1980). Considerada la relevancia que tienen para él, pueden constituirse tanto en una fuente importante de apoyo como en una de alteración de un desarrollo dentro de lo esperado.

Waters, Posada, Crowell y Lay (1994), evaluando la teoría del apego, una de las perspectivas de análisis de la construcción de los vínculos madre-niño más populares, mencionan que, luego de varias décadas de trabajo teórico y descriptivo, se sabe considerablemente poco sobre los mecanismos subyacentes de establecimiento y cambio en las relaciones tempranas de apego. Señalan que un paso en esta dirección podría ser la incorporación de los mecanismos tradicionales de aprendizaje, tanto para explicar el desarrollo de patrones específicos de apego como de su relación con otras variables psicológicas y conductuales concurrentes o futuras.

Como se aprecia, existe una importante cantidad de evidencia que apoya el supuesto de los significativos efectos de la relación del niño con su madre sobre el desarrollo emocional, conductual, social y cognitivo del niño.

Estos elementos marcan la importancia de desarrollar investigación acerca de la crianza, es decir, de los niveles en que las madres, durante la interacción con su niño, estimulan o retardan el desarrollo, así como de identificar los factores o variables que puedan estar influyendo en la calidad de esta estimulación.

Este estudio intenta realizar un acercamiento a los factores que conforman la crianza, que no constituye sino la más potente fuente de estimulación del desarrollo humano en la infancia, y que se supone sensible por lo menos a la cultura que contextualiza su despliegue.

Por otro lado, intenta, sobre la base de la identificación de esos factores, observar cómo el género del niño puede condicionar o modular la crianza recibida, considerando que esa eventual diferenciación tendrá un impacto sobre el desarrollo y la construcción de la identidad en la infancia, y en el desarrollo posterior.

$\mathrm{Si}$ deseamos crear una comunidad con menos brechas de oportunidades para niños y niñas, tenemos que empezar por identificar estas y cómo se producen; solo así resulta posible realizar propuestas que las aminoren o permitan una convivencia con condiciones de desarrollo más igualitarias.

En ese sentido, este estudio pretende responder al siguiente problema: ¿existen diferencias significativas en la crianza que brindan las madres según el género de su niño?

Utilizando una muestra de diadas madre-niño de San Juan de Lurigancho (Lima, Perú), se plantean los siguientes objetivos:

a) Identificar los factores de la crianza en el grupo señalado

b) Identificar si existen diferencias significativas tanto en los niveles de las 
dimensiones como en los factores de la crianza brindados por las madres de acuerdo con el género de su niño

Las hipótesis se plantean de la siguiente manera:

H1: Si comparamos los niveles en las dimensiones de la crianza brindados por un grupo de madres de acuerdo con el género de su niño, entonces hallaremos diferencias significativas entre ellos.

$\mathrm{H} 2$ : $\mathrm{Si}$ comparamos los niveles en los factores de la crianza brindados por un grupo de madres de acuerdo con el género de su niño, entonces hallaremos diferencias significativas entre ellos.

\section{LA ESTIMULACIÓN INFANTIL \\ EN LAS RELACIONES MADRE-NIÑO: FUNCIONES BÁSICAS}

Bueno (1997) menciona que cada uno de los eventos y objetos ambientales puede recibir el nombre de objeto-estímulo, el cual irradia continuamente energías diversas; a este proceso se le denomina estimulación. Pero también se puede denominar estímulos a los cambios discretos que acontecen en el ambiente, ya sea en los objetos-estímulo o en la estimulación.

Es evidente que, para este caso, caracterizar a una persona como un objeto-estímulo es, desde ya, una extrema simplificación. En el mismo sentido, Bijou y Baer (1980) afirman que, debido a que muchos estímulos de la madre varían dentro de amplios límites, es necesario considerarla no como una clase fija de estímulo, sino como un arreglo constantemente cambiante de estímulos de muchas clases.

Estos estímulos, que son muestras o segmentos de la estimulación que la madre otorga al niño, también son compartidos por otras personas.

Muchos estímulos brindados por la madre se hallan en cierta relación con las conductas del niño, a la cual llamaremos relación funcional si se constata que a una variación en la estimulación brindada por la madre sigue una variación en la conducta del niño.

Al prescribirse una relación funcional entre el estímulo brindado por la madre (segmento de su flujo de estimulación) y la conducta del niño, se puede hablar de una función-estímulo en esta relación: función-estímulo es un rótulo que indica la acción específica de aquel en la relación funcional que está siendo observada (Kantor y Smith, 1975).

En nuestro caso, las funciones-estímulo se concentran sobre las maneras en que los estímulos brindados por la madre controlan, producen, fortalecen, debilitan, etc., la conducta del niño.

Este concepto de función-estímulo sugiere el poder agrupar, al menos en dos categorías funcionales, muchos eventos o estímulos brindados por la madre, estímulos que promueven la adquisición y mantenimiento de conductas socialmente deseables, y estímulos que previenen, inhiben y extinguen conductas no deseables socialmente en el niño. Así se constituyen 
dos funciones básicas de la estimulación infantil: promover conductas y controlar conductas en el niño. Para efectos de este estudio, se considerarán ambas funciones como dimensiones de la crianza.

\section{La estimulación de promoción conductual}

La estimulación de promoción conductual es una cuya función es promover el aprendizaje de conductas socialmente deseables, que se refiere a un grupo de conductas-estímulo de la madre que tienen una probabilidad relativamente alta de generar el aprendizaje de conductas y habilidades socialmente deseables en el niño.

En ese sentido, el aislar las conductas identificadas con la estimulación de promoción conductual implicaría discriminar aquellas conductas-estímulo cuya función sería promover (a) la adquisición y (b) el mantenimiento de conductas adecuadas; y, por otro lado, promover la adquisición de habilidades propias del desarrollo, tales como (c) el autovalimiento y la independencia, así como (d) el aprendizaje de conductas verbales/sociales en el niño.

Adquirir conductas señala un proceso por el cual comportamientos que no se encontraban en el repertorio del niño ahora lo integran. Este proceso de adquisición va ganando probabilidades mediante el uso de determinados procedimientos que, en última instancia, implican una reorganización del ambiente del niño realizada por la madre y capaz de provocar la adquisición de estas nuevas conductas.
La reorganización puede implicar una variación en los estímulos físicos o colocar determinados objetos al alcance del niño; asimismo, puede ser una variación de índole social, como dar instrucciones simples o complejas, o brindar información espontáneamente; o una mezcla de ambas, es decir, la madre organiza tanto al ambiente como a sí misma para facilitar el aprendizaje; por ejemplo, puede dar modelos o enseñar progresivamente una conducta compleja, casos en los cuales, por lo general, se brindan múltiples estímulos en un arreglo único.

Asimismo, el mantenimiento de las conductas adquiridas, lo cual implica su uso o ejecución bajo condiciones en las que es esperado que sucedan, puede volverse probable si es que estas conductas obtienen una ventaja, beneficio o atención por su uso o ejecución en tales condiciones.

Es evidente que, cuando el niño nace, su repertorio de conductas es pequeño. Un indicador de desarrollo es, precisamente, la diversificación e incremento de conductas, así como cambios cualitativos en la forma de interacción con el entorno, producto, en gran parte, de las oportunidades que las personas de su ambiente le facilitan, que incluyen aquellas que promueven su independencia y autovalimiento (oportunidades de elegir, asumir responsabilidades y realizar actividades sin ayuda).

En resumen, la estimulación de promoción conductual en las relaciones madre-niño es el nivel de acción de la madre para promover conductas socialmente deseables en el repertorio del niño (Panduro, 1998a). 


\section{La estimulación de control conductual}

La estimulación de control conductual es un proceso cuya función es prevenir, inhibir y extinguir conductas no deseables en el niño; se refiere a un grupo de conductasestímulo de la madre con una probabilidad relativamente alta de controlar conductas inadecuadas en el niño. El desarrollo de estas se asocia (según datos de investigación), por un lado, a prácticas crueles de crianza e interacciones hostiles con el niño (Kingston y Prior, 1995); por otro, a la carencia de recursos en los padres para el manejo de ellas, tal como lo refieren, implícitamente, investigaciones como la de Lucano (1987), quien identificó entre las madres de niños hiperactivos niveles significativamente altos de actitudes de sobreprotección y sobreindulgencia en la relación con ellos. Otro trabajo importante es el de Feeham, McGee, Stanton y Silva (1991), quienes asociaron inconsistencia y bajos niveles de disciplina experimentados en la infancia con desórdenes de externalización o agresividad posteriores. Es preciso señalar que tanto las conductas inadecuadas del niño como las adecuadas están sujetas a los mismos principios del aprendizaje. Si obtienen beneficios o atención contingentes, particularmente de las personas significativas del ambiente, se genera una alta probabilidad para su mantenimiento en el repertorio del niño, y se incrementa su frecuencia e intensidad.

Así, el aislar las conductas identificadas con la estimulación de control conductual implicaría identificar aquellas conductas-estímulo cuya función sea controlar conductas inadecuadas en el niño: (a) anticipándose a su aparición, (b) inhibiéndolas cuando están presentes en el instante y (c) actuando de tal modo que, si sucedieron, su probabilidad futura de ocurrencia sea baja. En conclusión, la estimulación de control conductual en las relaciones madre-niño es el nivel de acción de la madre para controlar conductas no deseables socialmente en el repertorio del niño.

En este sentido, posee amplia importancia generar instrumentos que evalúen la calidad de esta relación, pues, según la evidencia, moldea significativamente el desarrollo presente del niño, y, al parecer, su futuro (Feeham, McGee, Stanton, y Silva, 1991).

\section{Investigaciones vinculadas al tema}

Entre las investigaciones cualitativas, se pueden mencionar los aportes de diversos investigadores.

Cabello, Ochoa y Filp (1992), en un estudio en una zona urbano-marginal de Santiago de Chile, hallaron que, aunque las madres suelen declarar que respecto del género no hacen diferencias cuando crían, durante las entrevistas con frecuencia mencionan (en forma velada o manifiesta) frases e ideas sobre conductas y actitudes que señalan lo contrario. Incluso en entrevistas con madres mayores, se identificó que la diferencia en la socialización de los niños y niñas fue establecida por ambos padres desde la infancia, incluso el nacimiento, y es trasmitida sutil e inconscientemente de manera cotidiana. Frases como las 
siguientes fueron identificadas como representativas de esta diferenciación:

"Es bien rebelde y eso que es mujer", "El niño hombre es más callejero", "Cuando ya empiezan a ser lolos, ahí hay que cuidar más a la mujer que al hombre. Porque a las niñas hay que evitar que vean cosas que se les graban".

González Ortiz (2005) describe los rituales que persisten a través del tiempo en el pueblo mazahua (México) para asignarle un género al sexo del recién nacido. Las diferencias que se convierten en desigualdades yacen desde el parto: si nace una niña, las parteras cobran menos que cuando nace un niño. Le continúa el enterramiento del cordón umbilical: el de las niñas se quema en el fogón, como símbolo del cuidado del hogar y del mantenimiento del fuego; el de los niños se entierra en el marco de la puerta de la casa o habitación que da al exterior y simboliza su papel de proveedor, cuyas actividades se encuentran fuera de la casa. En otras palabras, a las niñas se les destina el espacio privado y doméstico; a ellos, el espacio público y productivo.

Fuller (2000) señala que las desigualdades se manifiestan desde el valor que los padres conceden al tener un hijo o una hija. Un hijo representa una muestra de virilidad, el orgullo y la satisfacción de perpetuar un apellido, alguien que será el responsable de la familia, el heredero y demás. La hija representa a la persona a quien se tendrá que cuidar y a la que hay que casar "bien" para que un varón la cuide; los hombres desean tener hijas, siempre y cuando tengan, en primer lugar, un hijo.
Fuller (1997) halló, en un estudio previo realizado solo con padres en Lima, que los padres con hijos de ambos géneros en casa consideran que los varones deben conocerlo todo, tener libertad y ser, a diferencia de las mujeres, más abiertos. En cambio, aquellos que viven solo con hijas señalan que, si sus hijas fueran varones, podrían permitirles que fuesen más libres y mostrarles el mundo exterior. Al parecer, estos padres siguen reproduciendo el modelo de identidad de género que han aprendido, separando lo público de lo privado, estableciendo una división sexual del trabajo, e introduciendo a los varones al mundo de la calle y a las cualidades morales que han sido asociadas a la esfera pública.

Torres (2005) señala, como conclusión de un estudio en México con 35 padres de familia (varones) y con diferentes tipos de composición familiar, que la enseñanza y la formación que los varones reciben les ofrecen una realización completa, aunque con angustias y temores; les permiten probarse y probar el mundo externo, el dominio y el poder. En contraste, las condiciones que las mujeres reciben solo les hace posible vivir bajo el amparo y protección de un padre, un marido, un hijo; en fin, siempre de un varón que decida por ella, que la cuide, dirija, le enseñe qué hacer, sentir, pensar, hablar. Sin embargo, también les transmiten la imagen de un varón del que siempre deben desconfiar, temer, cuidarse, y del cual pueden esperar una traición. En la familia comienza la enseñanza de que hombres y mujeres no se relacionan: no pueden hacerlo porque son diferentes, toda vez que 
piensan y sienten distinto. Pareciera ser que, por el hecho de ser biológicamente disímiles, se generasen automáticamente diferencias psicológicas y sociales inmutables y permanentes. No se considera que tales diferencias, tal contraposición de hombres y mujeres, es una cuestión cultural más que natural; no se asume que se inician y evidencian en la familia, y que continúan en cada institución social con la que unos y otras tienen contacto.

Entre las investigaciones cuantitativas, se pueden considerar otros aportes.

Panduro (1998b), en un estudio con 320 diadas madre-niño de diferentes estratos de Lima, halló, respecto de la estimulación de promoción conductual en la crianza, que las madres tienden a estimular significativamente menos a los niños varones de 3 y 4 años, y mayor es la brecha a medida que se disminuye en estrato socioeconómico. Por otro lado, señala también que la estimulación de control conductual suele ser ejercida en un nivel significativamente mayor en las niñas, independientemente del estrato $y$ la edad del niño. Sostiene que esa diferencia en los niveles de control se asocia a una expectativa estereotipada acerca de la crianza de las niñas, y expectativas acerca del comportamiento correcto.

\section{Método}

\section{Participantes}

Se identificó un centro educativo inicial representativo del distrito de San Juan de Lurigancho, el CEI 0041 "El Bosquecito", situado en la Avenida Los Ciruelos s/n, Canto Grande, San Juan de Lurigancho, Lima. La población de este centro es mixta; tiene 250 alumnos matriculados al momento de la evaluación, distribuidos en los grados de Inicial 3 Años, Inicial 4 Años e Inicial 5 Años, y en dos turnos. Las edades de los niños oscilan entre los 4 y 7 años de edad. Todos los niños fueron evaluados, y la muestra final estuvo constituida por 241 niños (ver Tabla 1).

Según el INEI (2014), San Juan de Lurigancho es el distrito con mayor po-

\section{Tabla 1}

Distribución de la muestra según edad y género del niño

\begin{tabular}{cccc}
\hline & \multicolumn{3}{c}{ Género } \\
\cline { 2 - 3 } Edad & Masculino & Femenino & Total \\
\hline 4 & 17 & 19 & 36 \\
5 & 43 & 47 & 90 \\
6 & 47 & 43 & 90 \\
7 & 12 & 13 & 25 \\
Total & 119 & 122 & 241 \\
\hline
\end{tabular}


blación de Lima (1 069566 habitantes) y representa aproximadamente el $12 \%$ de la población de Lima Metropolitana.

Según el Plan de Desarrollo Concertado 2015-2021 (Municipalidad de San Juan de Lurigancho, 2014), la población en edad escolar es de 300000 personas aproximadamente. En general, su población es eminentemente joven: más del $53 \%$ es menor de 29 años. La pobreza se estima en 24 $\%$ (240 000 habitantes aproximadamente), lo que lo convierte en el distrito con mayor número de pobres en Lima en relación con otros: la pobreza extrema asciende al 0,8\% de la población. Sin embargo, su Índice de Desarrollo Humano Distrital es de 0.667, se ubica en el puesto 52 en el ranking distrital a nivel nacional y ha observado un progresivo incremento en dicho índice a lo largo de los últimos años. En 2012, alcanzó a exportar alrededor de 130 millones de dólares; gran parte de la PEA se desempeña en labores de comercio. El distrito tiene 1789 colegios, la mayoría privados (en primaria cuadriplican, y en secundaria triplican a los públicos); sin embargo, las pruebas censales en matemáticas y comprensión lectora reflejan que la calidad del rendimiento escolar está por debajo de muchos distritos de Lima. Un dato importante es que, para el año 2013, en el nivel de educación técnico-productivo, la mayoría de estudiantes fue mujer (55\% más que lo hombres). En general, la proporción de hombres y mujeres en los distintos niveles educativos es muy similar.

También es preciso señalar que el colegio escogido para realizar el estudio se ubica en la urbanización Canto
Rey, la cual constituye una zona con muchos matices de composición socioeconómica: en ella, se pueden encontrar sectores de pobreza hasta la clase media. Usualmente, la extrema pobreza de este distrito se halla en las zonas altas, en los cerros aledaños, en las periferias o en los límites con otros distritos. No es el caso de esta urbanización.

En general, se puede afirmar que la muestra constituye una representación de los matices sociales que se dan en la nueva Lima y en el distrito, poblaciones con fuertes aspiraciones hacia el progreso y con logros significativos en la productividad y prosperidad.

\section{Variables}

1. Género del niño de la diada (masculino y femenino)

2. Niveles en las dimensiones de la crianza (puntajes obtenidos en las subescalas de la Escala EEI)

3. Niveles en los factores de la crianza identificados (puntajes obtenidos en cada factor identificado en el análisis factorial practicado a cada una de las subescalas de la Escala EEI)

\section{INSTRUMENTOS}

Escala de Evaluación de la Estimulación Infantil en las Relaciones Madre-Niño: Escala EEI (Panduro, 1998a)

Permite identificar y medir en una escala continua el nivel de acción de la madre para promover conductas socialmente deseables y para controlar conductas no 
deseables socialmente en el repertorio de niños de 3 a 6 años. La Escala EEI se compone de 36 ítems, distribuidos de la siguiente manera: 15 ítems corresponden a la Subescala de Estimulación de Promoción Conductual (Subescala EPC), 14 ítems corresponden a la Subescala de Estimulación de Control Conductual (Subescala ECC) y 7 ítems corresponden a la Subescala de Mentiras (Subescala M).

La validez de contenido de las subescalas se estableció por el método de jueces. Las pruebas de validez de constructo señalaron correlaciones significativas $(\mathrm{p}<$. 001) en el análisis de ítems entre subescalas (Subescala EPC y ECC, $r=0.747$ ), y con medidas del desarrollo infantil (Subescala EPC y Escala de Madurez de Vineland, $\mathrm{r}=0.882$; y Subescala ECC y Lista de Chequeo de la Agresividad en Niños de Varona, $r=0.714$ ); correlaciones significativas $(\mathrm{p}<.05)$ también fueron identificadas en las pruebas de validez relacionada con criterios, para las cuales se utilizó la Escala de Actitudes hacia las Relaciones Madre-Niño de Roth (Subescala EPC y Sobreprotección, $r=-0.349$; Subescala ECC y Sobreindulgencia, $r=-0.365$ ). Por otro lado, las pruebas de confiabilidad indicaron coeficientes de estabilidad (Test-retest) de 0.886 y 0.746 , coeficientes de consistencia interna Spearman-Brown de 0.940 y 0.855 , y alpha de Cronbach de 0.910 y 0.789 para las Subescalas EPC y ECC, respectivamente.

\section{Ficha de datos de la diada}

Fue diseñada para obtener información básica sobre la diada madre-niño. Con respecto al niño, pidió información sobre su nombre, género, fecha y lugar de nacimiento, edad, número de hermanos y posición relativa entre ellos; con respecto a la madre, nombre, fecha y lugar de nacimiento, edad, estado civil, nivel de instrucción, ocupación, número de hijos incluyendo al niño y dirección.

\section{Procedimiento}

Se realizaron las coordinaciones con el colegio señalado para que las madres pudieran rendir, dentro de una semana definida, la evaluación en el colegio a la salida de clases del turno de estudios de sus hijos (mañana o tarde). Los materiales desarrollados fueron posteriormente depurados en función del nivel de mentiras. Luego, los datos fueron colocados en una base, que permitió establecer mediante análisis factorial los factores que conforman la crianza en este grupo, tanto en estimulación como en control del niño. Posteriormente se procedió a realizar las comparaciones respectivas de acuerdo con el género del niño mediante la Prueba $t$ para grupos independientes. Todos los resultados de los análisis estadísticos fueron valorados a un nivel de $p<.05$.

\section{Resultados}

\section{Análisis Factorial a las Subescalas de la Escala EEI o identificación de los factores de la crianza en la muestra}

La Tabla 2 muestra los resultados del análisis factorial aplicado a la Subescala de Promoción Conductual. Los 5 factores 
identificados explican el 53,77\% de la varianza. En cada columna, se han sombreado los ítems que formarán parte de cada factor. La Tabla 3 expresa los nombres asignados a los factores en función del concepto que se asume subyace a ellos.
La Tabla 4 muestra los resultados del análisis factorial aplicado a la Subescala de Control Conductual. Los 5 factores identificados explican el 53,14 \% de la varianza. En cada columna, se han sombreado los ítems que formarán parte de cada factor.

\section{Tabla 2}

Matriz de carga de Factores Rotados (método Varimax) para la Subescala de Promoción Conductual de la Escala EEI que explica el 53,77 \% de la varianza

\begin{tabular}{|c|c|c|c|c|c|}
\hline & Factc & & & & \\
\hline Ítem & 1 & 2 & 3 & 4 & 5 \\
\hline EI36P & .686 & & & & \\
\hline EI29P & .674 & & & & \\
\hline EI34P & .588 & & & & \\
\hline EI04P & .517 & & & & \\
\hline El19P & .513 & & & & \\
\hline El26P & .474 & & & & \\
\hline EI31P & & .739 & & & \\
\hline EI21P & & .581 & & & \\
\hline El16P & & .529 & & & \\
\hline EI09P & & & .847 & & \\
\hline El11P & & & .617 & & \\
\hline El14P & & & & .798 & \\
\hline EI06P & & & & .508 & \\
\hline EI01P & & & & .425 & \\
\hline El24P & & & & & .854 \\
\hline
\end{tabular}


Tabla 3

Factores identificados en el análisis factorial en la Subescala de Promoción Conductual

\begin{tabular}{ll}
\hline Factor & Ítems \\
\hline Factor 1: & $4,19,26,29,34,36$ \\
Receptividad e impulso al desarrollo & \\
\hline Factor 2: & $16,21,31$ \\
Promoción de la autonomía & 9,11 \\
\hline Factor 3: & \\
Enseñanza a prueba de fracasos & $1,6,14$ \\
\hline $\begin{array}{l}\text { Factor 4: } \\
\text { Modelado de formas de proceder y pensar }\end{array}$ & 24 \\
\hline Factor 5: & \\
Mediación de la socialización &
\end{tabular}

\section{Tabla 4}

Matriz de carga de Factores Rotados (método Varimax) para la Subescala de Control Conductual de la Escala EEI que explica 53,14\% de la varianza

\begin{tabular}{|c|c|c|c|c|c|}
\hline & & & Factor & & \\
\hline Ítem & 1 & 2 & 3 & 4 & 5 \\
\hline EI05C & .668 & & & & \\
\hline EI25C & .641 & & & & \\
\hline $\mathrm{EI} 20 \mathrm{C}$ & .558 & & & & \\
\hline EI02C & .527 & & & & \\
\hline EI32C & & .753 & & & \\
\hline EI30C & & .710 & & & \\
\hline El27C & & .577 & & & \\
\hline El17C & & & .785 & & \\
\hline El10C & & & .633 & & \\
\hline El15C & & & .363 & & \\
\hline El12C & & & & .673 & \\
\hline EI35C & & & & .563 & \\
\hline $\mathrm{EI} 22 \mathrm{C}$ & & & & & .413 \\
\hline EI07C & & & & & .581 \\
\hline
\end{tabular}

La Tabla 5 expresa los nombres asignados a los factores en función del concepto que se asume subyace a ellos.
Comparaciones en niveles de estimulación en las dimensiones y factores de la crianza brindada por la madre según el género del niño 


\section{Tabla 5}

Factores identificados en el análisis factorial en la Subescala de Control Conductual

\begin{tabular}{lc}
\hline \multicolumn{1}{c}{ Factor } & Ítems \\
\hline $\begin{array}{l}\text { Factor 1: } \\
\text { Prohibiciones y reprensión }\end{array}$ & $2,5,20,25$ \\
\hline Factor 2: & $27,30,32$ \\
Firmeza & $10,15,17$ \\
\hline Factor 3: & 12,35 \\
Uso de estrategias & \\
\hline Factor 4: & 7,22 \\
Coherencia & \\
\hline Factor 5: & \\
Empoderamiento &
\end{tabular}

Como se aprecia, solo se ha identificado una t significativa al $\mathrm{p}<.05$ en el factor Mediación de la socialización. En el resto de comparaciones, no se han identificado diferencias significativas.

La Figura 1 muestra los promedios de los niveles en el factor mediación de la socialización para niños y niñas a través de sus edades en un corte trasversal. Se observa que, independientemente de la edad, los niños experimentan mayores puntajes que las niñas.

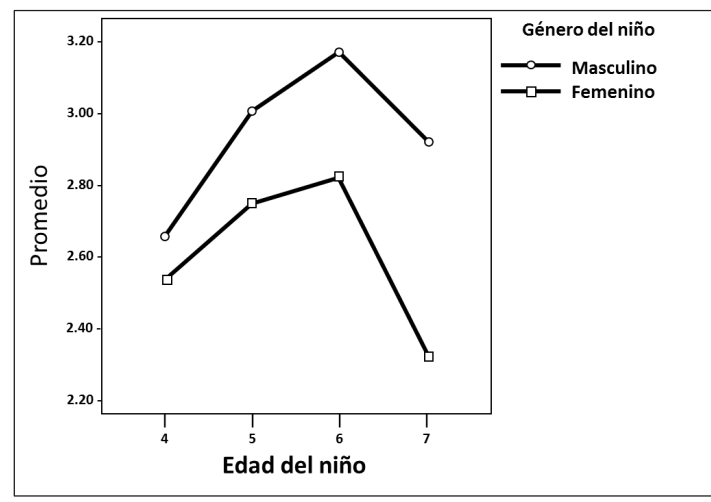

Figura 1. Mediación de la socialización para niños y niñas entre los 4 y 7 años 
Tabla 6

Comparación entre niños y niñas respecto de los niveles de Estimulación de Promoción y Control Conductual, así como en los factores brindados por sus madres en la crianza

\begin{tabular}{|c|c|c|c|c|c|c|c|}
\hline Dimensiones y factores & Género & $n$ & $M$ & $D T$ & $t$ & $p$ & $d_{\text {cohen }}$ \\
\hline \multirow{2}{*}{$\begin{array}{l}\text { Estimulación de } \\
\text { promoción conductual }\end{array}$} & Masculino & 119 & 43.08 & 7.19 & .058 & .954 & 0.001 \\
\hline & Femenino & 122 & 43.02 & 6.46 & & & \\
\hline \multirow{2}{*}{$\begin{array}{l}\text { 1. Receptividad } \\
\text { e impulso al } \\
\text { desarrollo }\end{array}$} & Masculino & 119 & 18.71 & 3.26 & -.305 & .761 & 0.004 \\
\hline & Femenino & 122 & 18.84 & 3.37 & & & \\
\hline \multirow{2}{*}{$\begin{array}{l}\text { 2. Promoción de la } \\
\text { autonomía }\end{array}$} & Masculino & 119 & 7.06 & 2.42 & -.627 & .531 & 0.01 \\
\hline & Femenino & 122 & 7.25 & 2.21 & & & \\
\hline \multirow{2}{*}{$\begin{array}{l}\text { 3. Enseñanza a } \\
\text { prueba de fracaso }\end{array}$} & Masculino & 119 & 5.89 & 1.47 & -.014 & .989 & 0.000 \\
\hline & Femenino & 122 & 5.89 & 1.60 & & & \\
\hline \multirow{2}{*}{$\begin{array}{l}\text { 4. Modelado de } \\
\text { formas de proceder } \\
\text { y pensar }\end{array}$} & Masculino & 119 & 8.87 & 2.20 & .181 & .857 & 0.002 \\
\hline & Femenino & 122 & 8.82 & 2.45 & & & \\
\hline \multirow{2}{*}{$\begin{array}{l}\text { 5. Mediación de la } \\
\text { socialización }\end{array}$} & Masculino & 119 & 3.01 & 1.03 & 2.412 & .017 & 0.03 \\
\hline & Femenino & 122 & 2.69 & 1.03 & & & \\
\hline \multirow{2}{*}{$\begin{array}{l}\text { Estimulación de control } \\
\text { conductual }\end{array}$} & Masculino & 119 & 32.11 & 5.93 & .178 & .859 & 0.002 \\
\hline & Femenino & 122 & 31.98 & 5.72 & & & \\
\hline \multirow{2}{*}{$\begin{array}{l}\text { 1. Prohibiciones y } \\
\text { reprensión }\end{array}$} & Masculino & 119 & 11.82 & 2.48 & .366 & .715 & 0.004 \\
\hline & Femenino & 122 & 11.70 & 2.55 & & & \\
\hline \multirow[t]{2}{*}{ 2. Firmeza } & Masculino & 119 & 5.71 & 2.66 & -.841 & .401 & 0.01 \\
\hline & Femenino & 122 & 5.99 & 2.47 & & & \\
\hline \multirow[t]{2}{*}{ 3. Uso de estrategias } & Masculino & 119 & 6.94 & 2.29 & .832 & .406 & 0.01 \\
\hline & Femenino & 122 & 6.70 & 2.27 & & & \\
\hline \multirow[t]{2}{*}{ 4. Coherencia } & Masculino & 119 & 4.25 & 1.58 & .154 & .878 & 0.002 \\
\hline & Femenino & 122 & 4.22 & 1.53 & & & \\
\hline \multirow[t]{2}{*}{ 5. Empoderamiento } & Masculino & 119 & 3.39 & 1.61 & .083 & .934 & 0.001 \\
\hline & Femenino & 122 & 3.37 & 1.72 & & & \\
\hline
\end{tabular}




\section{DISCUSIÓN}

Uno de los aportes de este estudio es la identificación de los factores de la crianza para esta muestra. El valor de este análisis radica en que responde a la necesidad de contar con instrumentos que permitan estudiar los vínculos de la madre con el niño desde las acciones concretas en la crianza, pero que también contemple los componentes al interior de las dimensiones consideradas.

Los resultados de las comparaciones de los factores de crianza señalan algunos hallazgos interesantes. Por un lado, el género no marca una diferencia sustancial en la crianza brindada por la madre, es decir, ni en la promoción ni en el control conductual de los niños. Esto coincide parcialmente con lo hallado en un estudio previo realizado por Panduro (1998b), en el cual se identificó que la estimulación de promoción conductual recibida no se vio afectada por el género del niño. Sin embargo, discrepa en cuanto a la estimulación de control; en el mismo estudio, sí se identificó que las niñas, independientemente del estrato socioeconómico, recibían mayores despliegues de disciplina. En el presente estudio, los análisis señalan que niños y niñas se ven expuestos a niveles similares de control por parte de sus madres. Esta variación puede deberse a la progresiva incorporación en la comunidad del concepto de que los niños y niñas deben acceder a similares condiciones de socialización y crianza, así como a similares despliegues de contención mediados por las madres.
Gran parte de las familias de la zona provienen de la sierra sur del país. La migración ha significado para ellas una oportunidad de replantear los conceptos tradicionales de crianza, en ese sentido, la incorporación y cercanía a la vida escolar (con los puntos de vista de la escuela, la relación con las maestras y la construcción de nuevas redes de soporte entre las madres [formales e informales]) pueden haber influenciado para que se produzca esta variación hacia la igualdad en el acceso a derechos y oportunidades de desarrollo para niños y niñas en general.

Este estudio no se orientó a identificar cuáles son las ideas asociadas a la crianza de niños y niñas. Lo hizo, más bien, a identificar los reportes acerca de las conductas concretas que despliegan las madres en la crianza. Cabello, Ochoa y Filp (1992), por ejemplo, identificaron en su estudio en una zona urbano marginal de Chile un discurso de igualdad, pero sostienen que era muy probable que los haceres fueran diferentes en la práctica. En el caso del presente estudio, se identifica, en general, que los haceres no son diferentes significativamente. Sería interesante plantear un estudio cualitativo acerca de las concepciones en torno a la crianza en función del género en este grupo, pues quizá suceda un fenómeno inverso al señalado.

Un aspecto sobre el que se sensibiliza en la escuela es la prevención de los problemas de conducta, concebidos como patrones de comportamientos disruptivos en la relación con las personas y el entorno: la contención y la estructura 
constituyen factores protectores frente a ellos y preventivos de situaciones graves futuras, como violencia y delincuencia, con sus respectivos matices.

Este distrito es uno de los más violentos de Lima: solamente en el 2013, hubo 11700 crímenes, de los cuales 6000 fueron contra el patrimonio y 1500 fueron homicidios o lesiones graves (INEI, 2014). Ante este panorama, el control desde el hogar puede ser concebido en esta comunidad como un elemento útil para la prevención de diversas dificultades sociales en los hijos.

Por otro lado, este estudio señala que, desde la infancia, las niñas son estimuladas al mismo nivel que los niños; ello se proyecta a lo largo del tiempo en, por ejemplo, la distribución porcentual muy similar en el acceso a los diversos niveles de educación, a excepción de la educación técnica, a la cual las mujeres en el distrito acceden en un porcentaje significativamente mayor que los hombres. Esto dista de la realidad de otras zonas del país. Este impulso moldea un estilo diferente de ser mujer, y parece estar asociado a una renovada concepción de la forma como se tiene que educar a niños y niñas desde la infancia. Hay un impulso hacia una mujer menos dependiente del hombre, que pueda valerse por sí misma y cuya vida no se restrinja a ser ama de casa, sino profesional o técnica, con autonomía económica. Parece ser que, en esta zona, la educación y la formación en el hogar son concebidas como los terrenos desde donde se pueden homogenizar las oportunidades para los hijos. Considerar que este distrito tiene más colegios privados que estatales sugiere la expectativa por acceder a una educación con mejores condiciones que las ofrecidas por el Estado, y en la cual vale invertir.

Cuando se realizaron las comparaciones entre niños y niñas por factores de la crianza, solo se identificó que se diferencian significativamente en el factor mediación de la socialización, perteneciente a la dimensión estimulación de promoción conductual. Este factor se refiere a aquellas acciones de la madre orientadas a facilitarle al niño el contacto social con otros niños y personas ("Le doy la oportunidad de relacionarse con diferentes niños y otras personas"). Las niñas tienen niveles significativamente menores que los niños en este factor; los niños experimentan una mayor mediación de este tipo que las niñas.

Esto podría señalar que las niñas son restringidas, en algún nivel, a vincularse solo con su familia, y los niños tendrían más oportunidad para explorar vínculos fuera de ella, incluso independientemente de la edad del niño. Es decir, constituye una pauta diferenciada que las madres desarrollan desde que los niños son pequeños. Esto hace a las niñas menos visibles en los entornos más allá de sus madres, comparadas con los niños. Por ejemplo, los niños, al estar ellos juntos, y desde muy pequeños, suelen armar clanes, algo así como asociaciones infantiles, donde compiten y toman (o se les asigna) un rol en la estructura de pares. Las niñas suelen llegar posteriormente a estas asociaciones, ya constituidas por 
varones, y en algunos casos solo como observadoras, por ejemplo, viendo a los niños jugar fútbol, relegadas a la periferia del patio escolar, la loza deportiva o la pista. Quizá sea la primera experiencia que muchas de ellas tendrán en la comunidad y la sociedad, en las cuales hallarán más estructuras constituidas por varones y preparadas solo para ellos, las cuales replicarán la exclusión o les otorgarán una condición de invisibilidad tanto a ellas físicamente como a sus derechos y necesidades.

Esta diferenciación en los niveles de mediación de la socialización también puede obedecer a la concepción de que "las niñas son para la casa y los niños para la calle", concepto que aparece en otros estudios cualitativos sobre crianza. Esa cercanía a la madre, o restricción en la mediación para la niña, puede entenderse en el marco de un interés por iniciar el moldeamiento progresivo al rol de mujer protectora y cuidadora en el hogar; tenerla cerca permite que este incipiente adoctrinamiento sea exitoso. No obstante, en muchos aspectos de la crianza, como los presentados en este estudio, hay exposición de los niños y las niñas a condiciones similares. Esta diferenciación puede estar basada en creencias que sostienen que los niños "requieren socializar" y las niñas menos, pues a ellas les "basta su mamá y su familia", un entorno de comodidad y protección. Asimismo, los efectos de esto sobre las habilidades sociales también se valoran diferencialmente: la niña tímida y hogareña es aceptable; el niño tímido y hogareño, no, porque "la niña es de su casa, el niño de la calle"; "la niña debe ser cuidada", porque "un hombre siempre cae parado".

La mediación o amplificación sistemática de los vínculos sociales puede promover un estilo de ser del niño: rasgos emocionales como la dependencia emocional en las niñas y la autonomía emocional, mayor seguridad ante las adversidades y los cambios en los niños. Las niñas, para insertarse, tienen que atenuar la potencia del vínculo con su familia y las expectativas de esta; tienen que hallar mecanismos y roles que les permitan existir o ser visibles, arreglárselas o negociar entre su familia y la productividad. Es interesante anotar que San Juan de Lurigancho, en 2013, se ubicó entre los dos distritos (de 49 que constituyen Lima y Callao) que más licencias municipales otorgó a panaderías, restaurantes, spas/peluquerías, farmacias y cabinas de internet donde, mayoritariamente, las mujeres son administradoras o pequeñas empresarias.

Actualmente, el Índice de Desigualdad de Género del Perú se ubica en un valor de 0,387 , lo cual refleja que nos encontramos en proceso de reducción de las desigualdades de género. Nos ubicamos muy cerca a Brasil. En el Perú, se ha avanzado más que en Paraguay, Nicaragua, Colombia, Honduras, República Dominicana, Panamá y Guatemala. En esta comunidad analizada, como se describió, las mujeres tienen una presencia proactiva en el funcionamiento económico: entre los años 1993 y 2007, la PEA de la mujeres subió de una tasa de actividad de 33,1 a 45,7 y la tasa anual de crecimiento en esos años fue el doble que la de los hombres 
(INEI, 2008). Según la Red de Salud del distrito, la tasa de embarazo adolescente se ha recortado a menos de la mitad del 2008 al 2012 (Municipalidad Distrital de San Juan de Lurigancho, 2014), y el nivel educativo es equiparable al de los varones. Incluso, como se mencionó, el acceso a formación técnica-productiva entre ellas es significativamente superior. Las mujeres y los varones van cerrando sus brechas de oportunidades, pero en este proceso las creencias estereotipadas acerca de los géneros quizá sean los aspectos más complejos de modificar. Sin embargo, las acciones están permitiendo reelaborar tales creencias.

Este estudio remarca la relevancia de trabajar por la desmitificación de las creencias y estereotipos asociados al género; contribuye con aminorar las desigualdades entre varones y mujeres.

La mediación y las oportunidades de socialización se generan en la infancia de las niñas desde las madres y la familia, y de hecho la escuela, en donde se deben construir las bases de la participación y visibilidad de ellas desde pequeñas. Contribuirán, además, inspirando positivamente la igualdad en diversos aspectos del desarrollo de las niñas, así como en una mejora sustancial del desarrollo humano global y sus indicadores.

Se puede afirmar que la evolución de los indicadores de crecimiento del distrito evidencia la presencia de una energía resiliente y una marcada orientación al logro entre las mujeres, expresadas en el trabajo, el progreso y la búsqueda de oportunidades a través de la educación y la empresa.
Sin embargo, en el escenario de esta comunidad, la violencia contra ellas es una amenaza aún por resolver, y de esta sí requieren ser protegidas. Quizá esa actitud de cuidado diferenciado y de restricción de la socialización se vea promovida por la expectativa de protegerlas de un contexto inmediato o futuro percibido como hostil, inseguro o peligroso. Un contexto así puede agudizar, justificar y normalizar actitudes de sobreprotección en el enfoque de crianza de los hijos en general; sin embargo, ello podría poner a las niñas en condición de mayores restricciones en la mediación de la socialización, y alimentar así su invisibilidad, nutrida esta última ya por los estereotipos de género.

En esta comunidad, se viene construyendo desde tiempo atrás, desde las acciones, un nuevo modelo de ser mujer. Se prevé que este significará un impulso imparable a través de las generaciones, pues las niñas criadas y educadas bajo estos ejemplos y aspiraciones es muy probable que repliquen en sus propias historias aquellos que sus madres mismas inspiraron a través de sus nuevas creencias, decisiones y modelos de logro.

El contraste de hipótesis permite afirmar lo siguiente:

- La comparación de los niveles en las dimensiones de la crianza brindada por un grupo de madres de acuerdo con el género de su niño no presenta diferencias estadísticas significativas.

- La comparación de los niveles en los factores de crianza brindados por un grupo de madres de acuerdo con el 
género de su niño determinó diferencias significativas entre ellos.

\section{REFERENCIAS}

Ato, E, Galián, M., y Huéscar, E. (2007). Relaciones entre estilos educativos, temperamento y ajuste social en la infancia: una revisión. Anales de Psicología, 23(1), 33-40.

Berk, L. (2004). Awakening Children's Minds: How Parents and Teachers Can Make a Difference. Oxford: University Press.

Bijou, S., y Baer, D. (1980). Psicología del desarrollo infantil: teoría empírica y sistémica de la conducta (Vol.1). México: Trillas.

Bueno, R. (1997). Introducción al campo interconductual. Psicología actual. Manuscrito aceptado para publicación.

Cabello, A., Ochoa, J., y Filp. J. (1992). Pautas y prácticas en la crianza de niños y niñas entre 0 y 6 años de sectores pobres. Santiago: CIDE-CELAM -UNICEF.

Cahill, K., Deater-Deckard, K., Pike, A., y Hughes, C. (2007). Theory of Mind, Self-Worth and the Mother-Child Relationship. Social Development, 16(1), 45-56.

Denham, S. (1993). Maternal Emotional ResponsivenessandToddlers'Social-EmotionalCompetence.JournalofChildPsychology and Psychiatry, 34, 715-728.

Esser, G., Dinter, R., Jorg, M., Rose, F., Villalba, P., Laucht, M., y Schmidt, M.
(1993). Importance and Determinants of Early Mother-Child Relations. Zeitschrift für Psychosomatische Medizin und Psychoanalyse, 39(3), 246-264.

Feeham, M., McGee, R., Stanton, W., y Silva, P. (1991). Strict and Inconsistent Discipline in Childhood: Consequences for Adolescent Mental Health. British Journal of Psychology, 30, 325-331.

Flanagan, P., Coppa, D., Riggs, S., y Alario, A. (1994). Comunication behaviors of enfants of teen mothers. An exploratory study. Journal of Adolescent Health, 15, 169-175.

Fuller, N. (Ed.) (2000). Paternidades en América Latina. Lima: Pontificia Universidad Católica del Perú/Fondo Editorial.

González, F. (2005). Mujeres que cuidan. Actos ritualizados y la construcción social de los géneros entre los mazahuas. En I. Vizcarra (Ed.), Género y poder, diferentes experiencias y mismas preocupaciones. México: Universidad Autónoma del Estado de México.

Henao, G., Ramírez, C., y Ramírez, L. (2007). Las prácticas educativas familiares como facilitadoras del proceso de desarrollo en el niño y niña. El Ágora, 7(2), 233-240.

INEI. (2008). Perfil sociodemográfico de la provincia de Lima. Lima: INEI.

INEI. (2014). Perú: Brechas de género, 2001-2013. Avances hacia la igualdad de mujeres y hombres. Lima: INEI.

INEI. (2014). Una mirada a Lima Metropolitana. Lima: INEI. 
Kantor, J., y Smith, N. (1975). The Science of Psychology: an Interbehavioral Survey. Chicago: Principia Press.

Kingston, L., y Prior, M. (1995). The Development of Pattern of Stable, Transient, and School-Age Onset Aggressive Behavior in Young Children. Journal of American Academy of Child and Adolescent Psychiatry, 34, 348- 358.

Kochanska, G., Forman, D., Aksan, N., y Dunbar, S. (2005). Pathways to Conscience: Early Mother-Child Mutually Responsive Orientation and Children's Moral Emotion, Conduct, and Cognition. Journal of Child Psychology and Psychiatry, 46(1), 19-34.

Lucano, C. (1987). Actitudes de un grupo de madres frente a sus hijos hiperactivos (Tesis de licenciatura). Universidad de San Martín de Porres, Lima.

Municipalidad Distrital de San Juan de Lurigancho. (2014). Plan de Desarrollo Concertado 2015-2021. Lima: Municipalidad de San Juan de Lurigancho.

Musselman, C., y Churchill, A. (1991). Conversational control inmother-childdyads.
Auditory-Oral versus Total Communication. American Annals of Deafness, 136, 5-16.

Panduro, J. (1998a). Evaluación funcional de las relaciones madre-niño: la Escala EEI. Revista de Psicología, 16(2), 241-264.

Panduro, J. (1998b). Estimulación infantil en las relaciones madre-niño. Teoría e Investigación en Psicología, 3(1), 151-176.

PNUD. (2014). Informe sobre desarrollo humano 2014. New York: PNUD.

Torres, L. (2005). Diferencias en la crianza paterna de tres grupos familiares. Enseñanza e Investigación en Psicologia, 10(1), 73-92.

Unicef. (2014). La escuela del silencio [Video]. Recuperado de https://www. youtube.com/watch? $\mathrm{v}=\mathrm{wBG}$ 3jUvTMCs

Waters, E., Posada, G., Crowell, J., y Lay, K. (1994). The Development of Attachment. From Control Systems to Working Models. Psychiatry, 57(1), 32-42. 


\section{ANEXO}

\section{EEI \\ Ps. José Antonio Panduro}

\section{INSTRUCCIONES:}

A continuación, encontrará un conjunto de afirmaciones acerca de cómo los padres y/o madres actúan con sus hijos. PENSANDO EN SU NIÑO(A), lea cada una de ellas y encierre en un círculo su respuesta de acuerdo con la frecuencia con la que a usted le sucedió durante los últimos SEIS MESES: nunca, rara vez, a veces, muchas veces o siempre.

Trate de ser SINCERO(A). Trabaje rápidamente y recuerde contestar todas las afirmaciones. Todas las respuestas son válidas.

Este material (EEI) lo recibirá por duplicado, pues debe ser desarrollado de manera individual por CADA PADRE Y POR SEPARADO, pensando en su niño(a).

Puede comenzar.

$$
\mathrm{N}=\text { Nunca } \quad \mathrm{R}=\text { Rara vez } \quad \mathrm{A}=\mathrm{A} \text { veces } \quad \mathrm{M}=\text { Muchas veces } \quad \mathrm{S}=\text { Siempre }
$$

\begin{tabular}{|l|l|l|l|l|l|l|}
\hline 1. & $\begin{array}{l}\text { Le explico cómo se puede beneficiar al hacer cosas positivas y } \\
\text { correctas. }\end{array}$ & $\mathrm{N}$ & $\mathrm{R}$ & $\mathrm{A}$ & $\mathrm{M}$ & $\mathrm{S}$ \\
\hline 2. & Le aviso anticipadamente lo que no debe hacer. & $\mathrm{N}$ & $\mathrm{R}$ & $\mathrm{A}$ & $\mathrm{M}$ & $\mathrm{S}$ \\
\hline 3. & Critico a otros padres de familia por la forma de criar a sus hijos. & $\mathrm{N}$ & $\mathrm{R}$ & $\mathrm{A}$ & $\mathrm{M}$ & $\mathrm{S}$ \\
\hline 4. & Lo aliento a enfrentar sus dificultades. & $\mathrm{N}$ & $\mathrm{R}$ & $\mathrm{A}$ & $\mathrm{M}$ & $\mathrm{S}$ \\
\hline 5. & Le explico por qué no debe hacer cosas negativas. & $\mathrm{N}$ & $\mathrm{R}$ & $\mathrm{A}$ & $\mathrm{M}$ & $\mathrm{S}$ \\
\hline 6. & $\begin{array}{l}\text { Le hago ver cómo hago las cosas para que después las haga de } \\
\text { esa manera. }\end{array}$ & $\mathrm{N}$ & $\mathrm{R}$ & $\mathrm{A}$ & $\mathrm{M}$ & $\mathrm{S}$ \\
\hline 7. & $\begin{array}{l}\text { Lo amenazo con el castigo que después le dará otra persona. } \\
\text { 8. }\end{array}$ & $\begin{array}{l}\text { Le he dicho "mentirillas". } \\
\text { Cuando le resulta difícil hacer algo, trato de que lo logre "paso a }\end{array}$ & $\mathrm{N}$ & $\mathrm{A}$ & $\mathrm{M}$ & $\mathrm{S}$ \\
\hline 9. & $\begin{array}{l}\text { Cuso" o "poco a poco". } \\
\text { paso }\end{array}$ & $\mathrm{M}$ & $\mathrm{S}$ \\
\hline 10. & $\begin{array}{l}\text { Cuando no quiere obedecer, le advierto que no hará o no tendrá } \\
\text { las cosas que más le gustan. }\end{array}$ & $\mathrm{N}$ & $\mathrm{R}$ & $\mathrm{A}$ & $\mathrm{M}$ & $\mathrm{S}$ \\
\hline
\end{tabular}




\begin{tabular}{|c|c|c|c|c|c|c|}
\hline 11. & $\begin{array}{l}\text { Le explico cómo hacer o lograr las cosas dándole } \\
\text { instrucciones ordenadas que pueda seguir fácilmente. }\end{array}$ & $\mathrm{N}$ & $\mathrm{R}$ & A & M & S \\
\hline 12. & $\begin{array}{l}\text { Cuando me doy cuenta de que está haciendo algo indebido, } \\
\text { le ordeno que pare de hacerlo. }\end{array}$ & $\mathrm{N}$ & $\mathrm{R}$ & A & M & S \\
\hline 13. & Soy puntual en sus horarios, "como un reloj". & $\mathrm{N}$ & $\mathrm{R}$ & A & M & S \\
\hline 14. & Le muestro o enseño cosas nuevas sin que me lo pida. & $\mathrm{N}$ & $\mathrm{R}$ & A & M & S \\
\hline 15. & $\begin{array}{l}\text { Cuando hace algo que me desagrada, lo ocupo en algo que } \\
\text { no le permita continuar lo que está haciendo. }\end{array}$ & $\mathrm{N}$ & $\mathrm{R}$ & A & M & S \\
\hline 16. & Le doy la oportunidad de tomar decisiones y llevarlas a cabo. & $\mathrm{N}$ & $\mathrm{R}$ & A & M & S \\
\hline 17. & Cuando se porta mal, le quito las cosas que más le gustan. & $\mathrm{N}$ & $\mathrm{R}$ & A & M & S \\
\hline 18. & Mis costumbres o hábitos de crianza son buenos y correctos. & $\mathrm{N}$ & $\mathrm{R}$ & A & M & S \\
\hline 19. & Le doy responsabilidades de acuerdo con su edad. & $\mathrm{N}$ & $\mathrm{R}$ & A & M & S \\
\hline 20. & $\begin{array}{l}\text { Cuando se ha portado mal, le hago saber lo incorrecto o } \\
\text { desagradable que fue su comportamiento. }\end{array}$ & $\mathrm{N}$ & $\mathrm{R}$ & A & M & S \\
\hline 21. & $\begin{array}{l}\text { Le doy la oportunidad de que haga por sí solo las cosas que } \\
\text { yo hago por él. }\end{array}$ & $\mathrm{N}$ & $\mathrm{R}$ & A & M & S \\
\hline 22. & Cumplo cuando le he advertido un castigo. & $\mathrm{N}$ & $\mathrm{R}$ & A & M & S \\
\hline 23. & Dejo de lado el cansancio y me doy un tiempo para él (ella). & $\mathrm{N}$ & $\mathrm{R}$ & A & M & S \\
\hline 24. & $\begin{array}{l}\text { Le doy la oportunidad de relacionarse con diferentes niños } \\
\text { y otras personas. }\end{array}$ & $\mathrm{N}$ & $\mathrm{R}$ & A & M & S \\
\hline 25. & $\begin{array}{l}\text { Lo reprendo o castigo inmediatamente después } \\
\text { de que se comporta mal. }\end{array}$ & $\mathrm{N}$ & $\mathrm{R}$ & A & M & S \\
\hline 26. & Presto atención cuando me habla. & $\mathrm{N}$ & $\mathrm{R}$ & A & M & S \\
\hline 27. & Cedo cuando me pide algo con insistencia. & $\mathrm{N}$ & $\mathrm{R}$ & A & M & S \\
\hline 28. & $\begin{array}{l}\text { Hace inmediatamente lo que le indico, sin refunfuñar } \\
\text { ni renegar. }\end{array}$ & $\mathrm{N}$ & $\mathrm{R}$ & A & M & S \\
\hline 29. & Doy respuestas a sus preguntas e inquietudes. & $\mathrm{N}$ & $\mathrm{R}$ & A & M & S \\
\hline 30. & Le ofrezco cosas para que deje de portarse mal. & $\mathrm{N}$ & $\mathrm{R}$ & $A$ & M & S \\
\hline
\end{tabular}




\begin{tabular}{|c|l|c|c|c|c|c|}
\hline 31. & $\begin{array}{l}\text { Inicio conversaciones con él y las mantengo } \\
\text { por un momento. }\end{array}$ & $\mathrm{N}$ & $\mathrm{R}$ & $\mathrm{A}$ & $\mathrm{M}$ & $\mathrm{S}$ \\
\hline 32. & Termino haciendo las cosas que le había ordenado hacer. & $\mathrm{N}$ & $\mathrm{R}$ & $\mathrm{A}$ & $\mathrm{M}$ & $\mathrm{S}$ \\
\hline 33. & Cuando no me siento bien, le respondo malhumorado(a). & $\mathrm{N}$ & $\mathrm{R}$ & $\mathrm{A}$ & $\mathrm{M}$ & $\mathrm{S}$ \\
\hline 34. & $\begin{array}{l}\text { Le doy mi completa atención cuando trata de enseñarme } \\
\text { algo nuevo que ha aprendido. }\end{array}$ & $\mathrm{N}$ & $\mathrm{R}$ & $\mathrm{A}$ & $\mathrm{M}$ & $\mathrm{S}$ \\
\hline 35. & Me río frente a él de sus travesuras o malcriadeces. & $\mathrm{N}$ & $\mathrm{R}$ & $\mathrm{A}$ & $\mathrm{M}$ & $\mathrm{S}$ \\
\hline 36. & Lo premio o felicito por las cosas positivas que hace. & $\mathrm{N}$ & $\mathrm{R}$ & $\mathrm{A}$ & $\mathrm{M}$ & $\mathrm{S}$ \\
\hline
\end{tabular}

